\title{
Low-temperature relaxation in the one-dimensional Ising model
}

\author{
J. Javier Brey and A. Prados \\ Física Teórica, Universidad de Sevilla, Apartado de Correos 1065, E-41080 Sevilla, Spain
}

(Received 16 August 1995)

\begin{abstract}
The decay of the spin-spin time correlation functions in a one-dimensional Ising model with Glauber dynamics is studied. In the low-temperature limit, an asymptotically valid continuous space equation is derived. It is a modified diffusion equation with a purely exponential relaxation term. Its solution leads to an exact Cole-Davison behavior of the spin autocorrelation in the frequency domain, while in the time description a Kohlrausch-Williams-Watts (KWW) function followed by an exponential decay is obtained. The exponent of the KWW function, analytically derived, is $1 / 2$, and not $\mathbf{0 . 6 3}$, as has been reported in the literature.
\end{abstract}

PACS number(s): 02.50.Ey, 05.50.+q, 77.22.Ch

\section{INTRODUCTION}

Nonexponential relaxation has atracted a lot of attention recently, in part motivated by the interest in explaining the origin of the slow relaxation observed in glasses and other complex systems [1]. An important result obtained is that nonexponential relaxation is also shown by systems with an apparent, very simple dynamics. In particular, relaxation in kinetic Ising models has been extensively studied $[2,3]$ and stretched exponential behavior has been found for different choices of the transition rates. Nevertheless, most of the works are numerical, and exact analytical results are scarce. This applies even to the case of one-dimensional systems with Glauber dynamics, which seem to be the simplest possibility.

One of the first observations of slow decay in Ising systems was reported by Anderson [4] and later by Bozdemir [5], both in the context of dielectric relaxation in polymers, and using Glauber's model [6]. They studied the frequency behavior of the complex permittivity, which is expressed in terms of the Fourier transform of the spin time autocorrelation funtion. By fitting numerically their results, they conclude that at low temperatures the complex permittivity approaches the Cole-Davidson function with an exponent $\beta_{\mathrm{CD}}=1 / 2$. The implications in time of a Cole-Davidson distribution in frequencies had been analyzed a few years before by Lindsey and Patterson, also using numerical techniques [7]. According to them, the above distribution is nearly equivalent to a stretched exponential or a Kohlrausch-Williams-Watts (KWW) function, and they gave the relationship between $\beta_{\mathrm{CD}}$ and the exponent $\beta_{\mathrm{KWW}} \cdot \beta_{\mathrm{KWW}}=0.63$ corresponds to the value $\beta_{\mathrm{CD}}=1 / 2$. Consequently, this has been the value used to characterize the relaxation of the spin autocorrelation function in the one-dimensional Ising model with Glauber dynamics [8-10].

The fact that dielectric relaxation and depolarized light scattering experiments in polymers lead, in general, to values of $\beta_{\mathrm{KWw}}$ smaller than 0.63 has stimulated the search of other dynamics for the Ising model that give smaller values of $\beta_{\mathrm{KWW}}[8,9,11]$. In some cases, the interactions have been substituted by local constraints on the dynamical events [12]. The objective was to try to make the dynamics of the system more cooperative, at least in the low-temperature limit. In this way, the value $\beta_{\mathrm{KWW}}=0.5$, or very close to it, has been repeatedly obtained.

In this work we consider once again the relaxation of the spin time autocorrelation function in the onedimensional Glauber model. There are several reasons for it. First, it is important to derive analytically as many results as possible in order to understand the physical mechanisms that are responsible for the relaxation of the system. For instance, we derive an evolution equation with two easily identifiable terms: one associated with diffusion and the other one with the creation of defects. Similar equations have been introduced as approximations to describe the evolution of some of the models mentioned above. Second, we wanted to verify the value $\beta_{\mathrm{KWW}}=0.63$ and see how the $\mathrm{KWW}$ function emerges from the equations. Our calculations show that the ColeDavidson distribution is asymptotically exact in the lowtemperature limit, with $\beta_{\mathrm{CD}}=1 / 2$. Nevertheless, in the time domain one does not obtain a single $\mathrm{KWW}$ function, but an exponential behavior appears in a time window where the relaxation is still relevant. Besides, the exponent of the $\mathrm{KWW}$ function describing most of the relaxation is $\beta_{\mathrm{KWW}}=1 / 2$, showing that the introduction of local constraints does not always increase the degree of cooperativity of the dynamics. Finally, we have previously studied the relaxation of the energy for the same model [10] and found it interesting to compare it with that of a different property.

The plan of the paper is as follows. The model is reviewed in Sec. II, where a general expression for the spin-spin time correlation functions is given. Section III contains the most relevant results. The low-temperature limit is analyzed and a continuous space equation for the time evolution of correlations is derived. From this equation the Cole-Davidson distribution is easily obtained. Then, relaxation in time domain is considered, leading to the identification of a $\mathrm{KWW}$ region at intermediate times. The initial part of the relaxation is the subject of Sec. IV. It is shown that, even though the decay taking 
place in it is negligible at low temperatures, it contains enough information to determine the value of the exponent $\beta_{\mathrm{KWW}}$. Section $\mathrm{V}$ is devoted to some final remarks.

\section{SPIN-SPIN TIME CORRELATION FUNCTIONS}

We consider an infinite one-dimensional Ising model. The state of the system is specified by the spin vector $\sigma=\left\{\sigma_{i}\right\}$, where $\sigma_{i}= \pm 1$ is the spin at site $i$. The energy of the system for a configuration $\sigma$ is

$$
\mathcal{H}(\sigma)=-J \sum_{i} \sigma_{i} \sigma_{i+1}
$$

with $J$ a positive constant. The evolution of the system is described by a Markov process with Glauber dynamics. Therefore, the conditional probability $p_{1 \mid 1}\left(\sigma, t \mid \sigma^{\prime}, t^{\prime}\right)$ of finding the system in the state $\sigma$ at time $t$, given it was in the state $\sigma^{\prime}$ at time $t^{\prime}, t^{\prime}<t$, obeys the master equation

$$
\begin{aligned}
\frac{\partial}{\partial t} p_{1 \mid 1}\left(\sigma, t \mid \sigma^{\prime}, t^{\prime}\right)= & \sum_{i=-\infty}^{\infty}\left[\omega_{i}\left(R_{i} \sigma\right) p_{1 \mid 1}\left(R_{i} \sigma, t \mid \sigma^{\prime}, t^{\prime}\right)\right. \\
& \left.-\omega_{i}(\sigma) p_{1 \mid 1}\left(\sigma, t \mid \sigma^{\prime}, t^{\prime}\right)\right]
\end{aligned}
$$

where $R_{i} \sigma$ is the configuration obtained from $\sigma$ by flipping the $i$ th spin, i.e., by changing $\sigma_{i}$ into $-\sigma_{i}$, and $\omega_{i}(\sigma)$ is the transition rate for the flip. The expression of this latter quantity is

$$
\omega_{i}(\sigma)=\frac{\alpha}{2}\left[1-\frac{\gamma}{2} \sigma_{i}\left(\sigma_{i-1}+\sigma_{i+1}\right)\right]
$$

Here $\alpha$ is another positive constant defining the time scale of the evolution of the system and $\gamma$ is a function of the temperature $T$ of the heat bath given by $\left(k_{B}\right.$ is Boltzmann's constant)

$$
\gamma=\tanh \frac{2 J}{k_{B} T}
$$

Our aim is to study the equilibrium time correlation function $\left\langle\sigma_{i}(0) \sigma_{i}(t)\right\rangle_{\mathrm{eq}}$. It is convenient to introduce the set of functions

$$
\begin{aligned}
A_{i j}(t) & =\left\langle\sigma_{i}(t) \sigma_{j}(0)\right\rangle_{\mathrm{eq}} \\
& =\sum_{\sigma} \sum_{\sigma^{\prime}} \sigma_{i} \sigma_{j}^{\prime} p_{1 \mid 1}\left(\sigma, t \mid \sigma^{\prime}, 0\right) p_{\mathrm{eq}}\left(\sigma^{\prime}\right),
\end{aligned}
$$

where the equilibrium distribution function of the system is

$$
p_{\mathrm{eq}}(\sigma)=\frac{e^{-\mathcal{H}(\sigma) / k_{B} T}}{\sum_{\sigma} e^{-\mathcal{H}(\sigma) / k_{B} T}}
$$

Time derivative of Eq. (5) yields

$$
\frac{\partial}{\partial t} A_{i j}(t)=-\alpha A_{i j}(t)+\frac{\alpha \gamma}{2}\left[A_{i-1, j}(t)+A_{i+1, j}(t)\right] .
$$

This hierarchy is to be solved with the initial condition

$$
A_{i j}(0)=\left\langle\sigma_{i} \sigma_{j}\right\rangle_{\mathrm{eq}}=\eta^{|i-j|}
$$

where we have introduced

$$
\eta=\tanh \frac{J}{k_{B} T}
$$

Upon deriving Eq. (8) we have used that $p_{1 \mid 1}\left(\sigma, t \mid \sigma^{\prime}, 0\right)=$ $\delta_{\sigma, \sigma^{\prime}}$. The form of Eq. (7) and the initial conditions given in Eq. (8) imply that $A_{i j}$ is a function of $|i-j|$. Thus we define functions $f_{n}(t)$ by

$$
f_{n}(t)=A_{i j}(t)=\left\langle\sigma_{j+n}(t) \sigma_{j}(0)\right\rangle_{\mathrm{eq}}
$$

with $n=i-j$. These functions obey the equations

$$
\frac{\partial}{\partial t} f_{n}=-\alpha f_{n}+\frac{\alpha \gamma}{2}\left(f_{n+1}+f_{n-1}\right)
$$

where $n$ is an integer in the range $-\infty<n<+\infty$. The above hierarchy, with the initial condition $f_{n}(0)=\eta^{|n|}$, can be easily solved by standard procedures, for instance, using the eigenfunctions method [13], and the result can be expressed as

$$
f_{n}(t)=\frac{1}{\pi} \frac{1-\eta^{2}}{1+\eta^{2}} \int_{0}^{\pi} d q \frac{\cos n q}{1-\gamma \cos q} e^{-\alpha(1-\gamma \cos q) t} .
$$

The one-dimensional Ising model we are dealing with has been used as a model for dielectric polarization [5]. Under certain simplifying hypotheses, the complex permittivity $\epsilon$ was shown to be related to $f_{0}(t)$ through the relation

$$
\begin{aligned}
\epsilon^{*}(\omega) \equiv \frac{\epsilon(\omega)-\epsilon_{\infty}}{\epsilon_{0}-\epsilon_{\infty}} & =\int_{0}^{\infty} d t e^{-i \omega t}\left(-\frac{\partial}{\partial t} f_{0}(t)\right) \\
& =1-i \omega \int_{0}^{\infty} d t e^{-i \omega t} f_{0}(t)
\end{aligned}
$$

where $\epsilon_{0}$ and $\epsilon_{\infty}$ are the limiting low- and high-frequency permittivities, respectively. From Eq. (12) we have

$$
\begin{aligned}
-\frac{\partial}{\partial t} f_{0}(t) & =\frac{\alpha}{\pi} \frac{1-\eta^{2}}{1+\eta^{2}} \int_{0}^{\pi} d q e^{-\alpha(1-\gamma \cos q) t} \\
& =\alpha \frac{1-\eta^{2}}{1+\eta^{2}} e^{-\alpha t} I_{0}(\alpha \gamma t) .
\end{aligned}
$$

Here $I_{0}$ is the zeroth-order modified Bessel function. Substitution into Eq. (13) yields

$$
\epsilon^{*}(\omega)=\alpha \frac{1-\eta^{2}}{1+\eta^{2}} \frac{1}{\left[(i \omega+\alpha)^{2}-\alpha^{2} \gamma^{2}\right]^{1 / 2}} .
$$

Bozdemir [5] has also shown numerically that this expression approaches at low temperatures the Cole-Davidson distribution

$$
\epsilon^{*}(\omega)=\frac{1}{\left(1+i \omega \tau_{\mathrm{CD}}\right)^{\beta_{\mathrm{CD}}}}
$$

where $\tau_{\mathrm{CD}}$ is a constant and $\beta_{\mathrm{CD}}=1 / 2$. It must be pointed out that Anderson [4] had previously observed 
the same behavior in an equivalent model, although in neither of the two references is a detailed analytical derivation of Eq. (16) presented.

Lindsey and Patterson have carried out an extensive numerical study about the relation between the two functions $f_{0}(t)$ and $\epsilon^{*}(w)$ and concluded that corresponding approximately to a Cole-Davidson function in the frequency domain is a KWW or stretched exponential function for $f_{0}(t)[7]$, i.e.,

$$
f_{0}(t)=\exp \left[-\left(t / \tau_{\mathrm{KWW}}\right)\right]^{\beta_{\mathrm{KWw}}} .
$$

They also give an expression, obtained by fitting the numerical data, to obtain $\beta_{\mathrm{KWw}}$ from $\beta_{\mathrm{CD}}$. In particular, for $\beta_{\mathrm{CD}}=1 / 2$ their expression gives $\beta_{\mathrm{KWW}} \simeq 0.63$. This latter value has been used often in the literature to compare the relaxation spectrum of Ising models with different dynamics $[8,9]$ and also to claim that different properties of the Ising model with a given dynamics relax with different values of $\beta_{\mathrm{KWW}}$ (in the low-temperature limit) [10]. Here it will be shown that, at low temperatures, the function $f_{0}(t)$ is actually accurately described by a $\mathrm{KWW}$ function over a time window that contains most of the relevant part of the relaxation, but the value of the exponent is $\beta_{\mathrm{KWW}}=1 / 2$.

\section{THE LOW-TEMPERATURE LIMIT}

To clearly understand what happens at low temperatures it is convenient to establish first some general features of the function $f_{0}(t)$. From Eq. (12) and by means of a simple change of variable we can write

$$
f_{0}(t)=\int_{\lambda_{1}}^{\lambda_{2}} d \lambda g(\lambda) e^{-\lambda t}
$$

where the relaxation spectrum $g(\lambda)$ is

$$
g(\lambda)=\frac{\alpha}{\pi} \frac{1-\eta^{2}}{1+\eta^{2}} \frac{1}{\left[\left(\lambda-\lambda_{1}\right)\left(\lambda_{2}-\lambda\right)\right]^{1 / 2} \lambda}
$$

and $\lambda_{1}=\alpha(1-\gamma)$ and $\lambda_{2}=\alpha(1+\gamma)$ are the minimum and maximum relaxation rates, respectively. As a consequence of the inicial condition $f_{0}(0)=1$, the distribution $g(\lambda)$ is normalized to one. The average frequency is

$\lambda_{M}=\int_{\lambda_{1}}^{\lambda_{2}} d \lambda \lambda g(\lambda)=-\left[\frac{\partial}{\partial t} f_{0}(t)\right]_{t=0}=\alpha \frac{1-\eta^{2}}{1+\eta^{2}}$.

The short time behavior of $f_{0}(t)$ is now easily obtained

$$
\begin{aligned}
f_{0}(t)= & e^{-\lambda_{M} t} \int_{\lambda_{1}}^{\lambda_{2}} d \lambda g(\lambda) e^{-\left(\lambda-\lambda_{M}\right) t} \\
= & e^{-\lambda_{M} t} \int_{\lambda_{1}}^{\lambda_{2}} d \lambda g(\lambda)\left[1-\left(\lambda-\lambda_{M}\right) t\right. \\
& \left.+\frac{1}{2}\left(\lambda-\lambda_{M}\right)^{2} t^{2}+\cdots\right] \\
= & e^{-\lambda_{M} t}\left[1+O\left(\mu^{2} t^{2}\right)\right],
\end{aligned}
$$

i.e., the initial relaxation is exponential. The width $\mu$ of the relaxation spectrum is defined as

$$
\mu^{2}=\int_{\lambda_{1}}^{\lambda_{2}} d \lambda\left(\lambda-\lambda_{M}\right)^{2} g(\lambda)
$$

For the long time limit, the asymptotic behavior of Eq. (18) is

$$
f_{0}(t) \sim\left(\frac{1+\gamma}{2 \pi}\right)^{1 / 2} \frac{e^{-\lambda_{1} t}}{\left(\lambda_{1} t\right)^{1 / 2}}
$$

i.e., again an essentially exponential behavior, but in this case with a relaxation frequency $\lambda_{1}$. In the hightemperature limit, the relaxation spectrum is very narrow $\mu \ll \lambda_{M}$ and the relaxation is dominated by the initial exponential Eq. (21).

Consider now the low-temperature limit, in which $\gamma(T)$ approaches one from below. To study it we introduce the parameter

$$
\epsilon=1-\gamma
$$

and take the limit $\epsilon \ll 1$, which yields

$$
\begin{gathered}
\eta=1-(2 \epsilon)^{1 / 2}+O(\epsilon), \\
\lambda_{M} \sim \alpha(2 \epsilon)^{1 / 2},
\end{gathered}
$$

and

$$
\lambda_{1}=\alpha \epsilon .
$$

Therefore, in this limit $\lambda_{1} \ll \lambda_{M}$ and a clear separation of time scales shows up. As a consequence, there must be an intermediate time region in which the decay is highly nonexponential [3]. We can get more information by considering the average relaxation time $\langle\tau\rangle$ defined as

$$
\langle\tau\rangle=\int_{0}^{\infty} d t f_{0}(t)
$$

It is easily seen that this quantity diverges as $\epsilon^{-1}$ for $\epsilon \rightarrow 0$. Taking into account that the initial relaxation corresponds to an average relaxation time $\lambda_{M}^{-1}$, which goes as $\epsilon^{-1 / 2}$, we conclude that the initial exponential decay becomes irrelevant in the low-temperature limit.

Although it is, of course, possible to study also the behavior of $f_{0}(t)$ at intermediate times using its explicit expression given by Eq. (18), we will follow a different approach. There are several reasons for this choice. First, the method we will employ can be useful to study other models for which the exact expression of the relaxation function to be investigated is not known. Second, through our calculations we will obtain an equation for the correlations, valid in the low-temperature limit. Similar equations have been introduced without a solid justification to describe low-temperature relaxation in other models. A discussion of such equations in the light of what happens in our case is clarifying. Finally, our approach leads to a direct identification of the mechanisms responsible for the relaxation of the system and to the reason why most of the relevant part of the relaxation is nonexponential. 
Consider again Eq. (11), but now written in the form $\frac{\partial}{\partial t} f_{n}(t)=-\alpha\left(f_{n}-\frac{1}{2} f_{n+1}-\frac{1}{2} f_{n-1}\right)-\frac{\alpha \epsilon}{2}\left(f_{n+1}+f_{n-1}\right)$,

with the initial condition

$$
f_{n}(0)=\left[1-(2 \epsilon)^{1 / 2}+O(\epsilon)\right]^{|n|} .
$$

A perturbation expansion of the above hierarchy in powers of $\epsilon^{1 / 2}$ is singular because $f_{n}(t)$ is bounded by one for all $t$, while the terms of the expansion of $f_{n}(0)$ diverge for $|n| \rightarrow \infty$. This leads us to scale the variable $n$, so that the terms of the expansion of $f_{n}(0)$ remain finite, even in the limit $|n| \rightarrow \infty$. The scaling factor emerges quite naturally for the following reasons. For $t=0$ the time correlation function becomes the equilibrium space correlation function. The correlation length is defined by

$$
f_{n}(0)=e^{-|n| / \xi},
$$

and comparing with the expression of $f_{n}(0)$ we get

$$
\xi=\frac{1}{|\ln \eta|}
$$

In the low-temperature limit $\xi$ diverges, suggesting that it defines the relevant length scale. Since $|\ln \eta| \sim(2 \epsilon)^{1 / 2}$, we introduce the scaled length $x$ as

$$
x=n(2 \epsilon)^{1 / 2} \text {. }
$$

In terms of this variable the hierarchy given by Eq. (29) reads

$$
\begin{aligned}
\frac{\partial}{\partial t} f(x, t)= & -\alpha\left[f(x, t)-\frac{1}{2} f\left(x+(2 \epsilon)^{1 / 2}, t\right)\right. \\
& \left.-\frac{1}{2} f\left(x-(2 \epsilon)^{1 / 2}, t\right)\right] \\
& -\frac{\alpha \epsilon}{2}\left[f\left(x+(2 \epsilon)^{1 / 2}, t\right)+f\left(x-(2 \epsilon)^{1 / 2}, t\right)\right],
\end{aligned}
$$

where $f(x, t)=f_{n}(t)$. Now we expand the series and neglect terms of order $\epsilon^{2}$. The result is

$$
\frac{\partial}{\partial t} f(x, t)=\alpha \epsilon \frac{\partial^{2}}{\partial x^{2}} f(x, t)-\alpha \epsilon f(x, t)
$$

or, dehning also a time scale by

$$
\begin{gathered}
s=\alpha \epsilon t \\
\frac{\partial}{\partial s} f(x, s)=-f(x, s)+\frac{\partial^{2}}{\partial x^{2}} f(x, s) .
\end{gathered}
$$

The initial condition for this equation is

$$
f(x, 0)=e^{-|x|} .
$$

In this equation the two mechanisms responsible for the relaxation at low temperatures show up clearly in an ad- ditive way. There is a pure relaxation term, describing an exponential relaxation, and a diffusive term. An example of simple processes corresponding to each of the two mechanisms is given in Fig. 1. Notice that the definition of the time scale $s$ implies that the relevant times correspond to $t \sim(\alpha \epsilon)^{-1}$. Therefore, Eq. (37) does not describe the initial exponential relaxation, which, as we saw, takes place over times of order $\epsilon^{-1 / 2}$. Nevertheless, we also know that at very low temperatures initial decay is not relevant, in the sense that the correlation function remains practically constant and equal to its initial value. The solution of Eq. (37) is

$$
f(x, s)=\frac{2}{\pi} \int_{0}^{\infty} d k \frac{\cos k x}{1+k^{2}} e^{-\left(1+k^{2}\right) s}
$$

and thus the spin autocorrelation function is

$$
f_{0}(s) \equiv f(0, s)=\frac{2}{\pi} \int_{0}^{\infty} d k \frac{1}{1+k^{2}} e^{-\left(1+k^{2}\right) s} .
$$

Use of this expression in Eq. (13) gives, for the permittivity in the low-temperature limit,

$$
\epsilon^{*}(\omega)=\frac{1}{(1+i \hat{\omega})^{1 / 2}},
$$

where we have introduced

$$
\hat{\omega}=\frac{\omega}{\alpha \epsilon} .
$$

Therefore, we have derived the Cole-Davidson distribution with $\beta_{\mathrm{CD}}=1 / 2$, in agreement with previous numerical calculations $[4,5]$. The characteristic relaxation time is $\tau_{\mathrm{CD}}=(\alpha \epsilon)^{-1}$, which is of order unity over the relevant time scale $s$. The long time limit of Eq. (40) is

$$
f(0, s) \sim \frac{e^{-s}}{(\pi s)^{1 / 2}}
$$

which coincides with the low-temperature limit of Eq. (23). On the other hand, Eq. (40) does not present an initial exponential decay for the reasons discussed above. The limit $s \ll 1$ corresponds to an intermediate time region in which the "slow" time scale $s$ is small, but the real time $t$ is large. This is precisely the time window that must be analyzed to look for the nonexponential

(a)

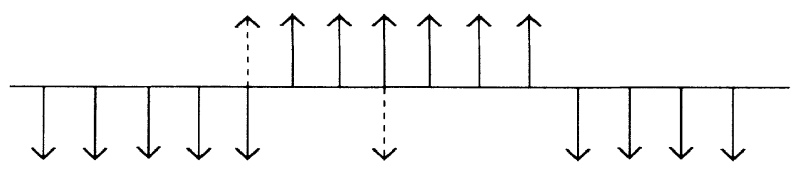

(b)

FIG. 1. Sketch of simple processes corresponding to the two mechanisms of relaxation at low temperatures described by Eq. (37): (a) the diffusive term and (b) the purely exponential relaxation. 
relaxation. To do so, we rewrite Eq. (40) as

$$
f(0, s)=1-\frac{1}{\pi^{1 / 2}} \int_{0}^{s} d u u^{-1 / 2} e^{-u}
$$

which for $s \ll 1$ can be approximated by

$$
\ln f(0, s) \simeq-\frac{1}{\pi^{1 / 2}} \int_{0}^{s} d u u^{-1 / 2} e^{-u} \simeq-\frac{2}{\pi^{1 / 2}} s^{1 / 2}
$$

or, returning to the $t$ variable,

$$
\ln f(0, t) \simeq-\left(\frac{4 \alpha \epsilon t}{\pi}\right)^{1 / 2}
$$

This expression holds for $\alpha t \ll \epsilon^{-1}$. By putting together the results we have obtained, we arrive at a description of the relaxation of the spin autocorrelation function through three well defined time regimes:

$$
f(0, t)= \begin{cases}\exp \left[-(2 \epsilon)^{1 / 2} \alpha t\right] & \text { for } \alpha t \ll 1 \\ \exp \left[-(4 \alpha \epsilon t / \pi)^{1 / 2}\right] & \text { for } 1 \ll \alpha t \ll \epsilon^{-1} \\ (\pi \alpha \epsilon t)^{-1 / 2} \exp (-\alpha \epsilon t) & \text { for } \alpha t \gg \epsilon^{-1}\end{cases}
$$

This behavior is similar to the one found in Ref. [10] for the linear relaxation of the energy following an homogeneous temperature perturbation, also for the Ising model with Glauber dynamics. The only relevant difference is that the prefactor of the exponential in the long time regime goes, in the case of the energy, as $t^{-3 / 2}$. Moreover, Shore and Zwanzig [14] have obtained the same three temporal regimes in a one-dimensional lattice model.

Thus we have shown that at intermediates times $f_{0}(t)$ is described by a stretched exponential function with $\beta_{\mathrm{KWW}}=1 / 2$. Note that this is an exact result, valid in the asymptotic limit of low temperatures. An example is given in Fig. 2, where $f_{0}(t)$ is plotted for $\epsilon=10^{-4}$. The solid line is the KWW function given by Eq. (46). We can estimate roughly the time interval in which the KWW function gives an accurate description of $f_{0}(t)$ by considering that it is bounded by its intersections $t_{1}$ and $t_{2}$ with the initial and the final exponentials. From Eq. (47) one gets $t_{1}=2 / \alpha \pi$ and $t_{2} \simeq 0.56 / \alpha \epsilon$. The correlation function at these two times takes the values $f\left(0, t_{1}\right)=\exp \left[-2^{3 / 2} \epsilon^{1 / 2} / \pi\right]$ and $f\left(0, t_{2}\right) \simeq 0.42$, respectively. If we consider, for instance, a temperature corresponding to $\epsilon=10^{-4}$, it is $f\left(0, t_{1}\right) \simeq 0.99$, showing that the initial exponential decay becomes negligible in the low-temperature limit. On the other hand, the value of $f\left(0, t_{2}\right)$ does not depend on the temperature and there is no reason to expect the $\mathrm{KWW}$ function to give an accurate description of the relaxation for values of $f(0, t)$ smaller than 0.4. Between this value and the asymptotic exponential decay at long times the apparent value of $\beta_{\mathrm{KWW}}$ increases from 0.5 to 1 . Of course, one can force the complete relaxation to be fitted by a unique KWW function, then getting a value of $\beta_{\mathrm{KWW}}$ in the above interval. This is the way in which Lindsey and Petterson obtained numerically the value $\beta_{\mathrm{KWW}} \simeq 0.63$. Although it is clear that this value gives some information about the

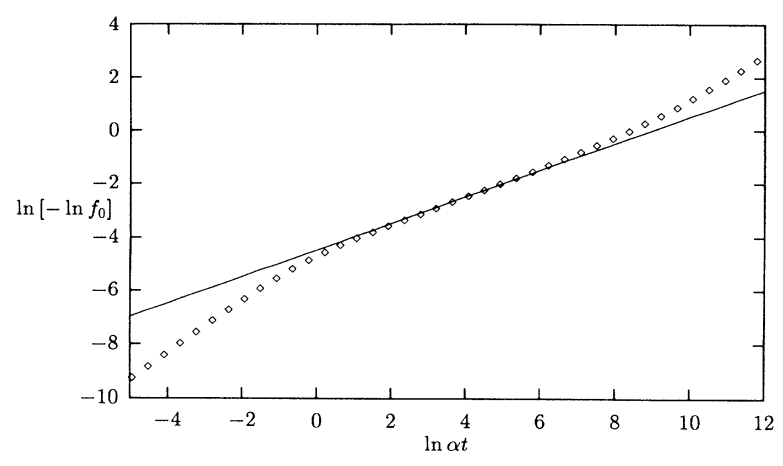

FIG. 2. Plot of the equilibrium spin autocorrelation function for a temperature value corresponding to $\epsilon=10^{-4}$. The diamonds are the numerical integration of Eq. (18) and the solid line is the KWW function of Eq. (46). Note that the logarithm scale amplifies the discrepancies, especially for short times where the difference between the exact solution and the $\mathrm{KWW}$ function is in fact negligible, as discussed in the text.

average relaxation of $f(0, t)$, one cannot conclude from it that the relaxation is less cooperative (the spectrum narrower) in this model than in other systems for which a fitting of the data gives $\beta_{\mathrm{KWW}} \simeq 0.5$.

\section{THE INITIAL PART OF THE RELAXATION}

Although the results in the preceding section lead to a complete description of the relaxation of the equilibrium spin autocorrelation function at low temperatures, it is interesting to consider an alternative analysis, which is valid in the short time regime. In fact, the procedure we will follow here is also appropriate for those models in which the final exponential relaxation either does not exist or is irrelevant. This seems to be the case, for instance, if a local constraint allowing only flips conserving the energy is introduced in the model we are considering $[8,9,11]$.

As discussed below Eqs. (29) and (30), they are not useful, in their present form, to carry out an expansion in powers of $\epsilon^{1 / 2}$. However, the difficulty is easily surpassed by going to the hierarchy for the time derivatives

$$
q_{n}(t) \equiv \frac{\partial}{\partial t} f_{n}(t)
$$

They obey the same hierarchy as $f_{n}$, i.e.,

$$
\frac{\partial}{\partial t} q_{n}=-\alpha\left(q_{n}-\frac{1}{2} q_{n-1}-\frac{1}{2} q_{n+1}\right)-\frac{\alpha \epsilon}{2}\left(q_{n-1}+q_{n+1}\right),
$$

but the initial condition is different. A simple calculation gives

$$
q_{n}(0)=\left[-\alpha(2 \epsilon)^{1 / 2}+O(\alpha \epsilon)\right] \delta_{n 0},
$$

where $\delta$ is the Kronecker function. Since the terms in the expansion of $q_{n}(0)$ are bounded for all $n$, we can now obtain a series expansion solution of the hierarchy given 
by Eq. (49). We write

$$
q_{n}(t)=-\alpha(2 \epsilon)^{1 / 2} \zeta_{n}(t)+O(\alpha \epsilon) .
$$

Substitution into Eq. (49) leads to

$$
\frac{\partial}{\partial t} \zeta_{n}=-\alpha\left(\zeta_{n}-\frac{1}{2} \zeta_{n-1}-\frac{1}{2} \zeta_{n+1}\right),
$$

to be solved with the initial condition

$$
\zeta_{n}(0)=\delta_{n 0}
$$

Thus $\zeta_{n}$ obeys a symmetric random walk master equation with the particle located initially at the origin. The solution of the equation is [13]

$$
\zeta_{n}(t)=\frac{1}{\pi} \int_{0}^{\pi} d q \cos n q e^{-\alpha t(1-\cos q)},
$$

and introduction of this into Eq. (51) yields

$$
q_{n}(t)=-\alpha(2 \epsilon)^{1 / 2} e^{-\alpha t} I_{n}(\alpha t)+O(\alpha \epsilon),
$$

where $I_{n}(t)$ is the $n$ th-order modified Bessel function. From this result we have

$$
\begin{aligned}
f_{0}(t) & =f_{0}(0)+\int_{0}^{t} d t^{\prime} q_{0}\left(t^{\prime}\right) \\
& =1-(2 \epsilon)^{1 / 2} \alpha t e^{-\alpha t}\left[I_{0}(\alpha t)+I_{1}(\alpha t)\right]+O(\alpha \epsilon t) .
\end{aligned}
$$

Neglecting $O(\alpha \epsilon t)$ in this expression is not correct for all times. In particular, the result does not verify $\lim _{t \rightarrow \infty} f_{0}(t)=0$. Only in the limit $\alpha \epsilon t \ll 1$ can we approximate

$$
\ln f_{0}(t) \simeq-(2 \epsilon)^{1 / 2} \alpha t e^{-\alpha t}\left[I_{0}(\alpha t)+I_{1}(\alpha t)\right]
$$

This equation can be shown to be equivalent to the one reported by Budmir and Skinner for the constant energy Ising model [15]. If we now consider here $\alpha t \ll 1$, we recover once again the initial exponential relaxation of Eqs. (21) and (26), while for $\epsilon^{-1} \gg \alpha t \gg 1$, the KWW decay of Eq. (46) is found.

Although Eq. (57) reproduces both the initial exponential decay and the KWW intermediate function, it is appropriate to underline that it is not able to describe most of the relevant part of the relaxation, which takes place on a time scale for which that expression is not valid. Of course, a modification of the dynamics of the system can lead to a change of the relevant time scale in such a way that the final exponential decay is negligible. Then the relaxation is accurately given by Eq. (57). This may be the case in Refs. $[9,11]$.

The analysis in this section has another interesting consequence. Let us define an effective time-dependent KWW exponent by

$$
\beta_{\text {eff }}(t)=\frac{\partial}{\partial \ln t} \ln \left[-\ln f_{0}(t)\right] .
$$

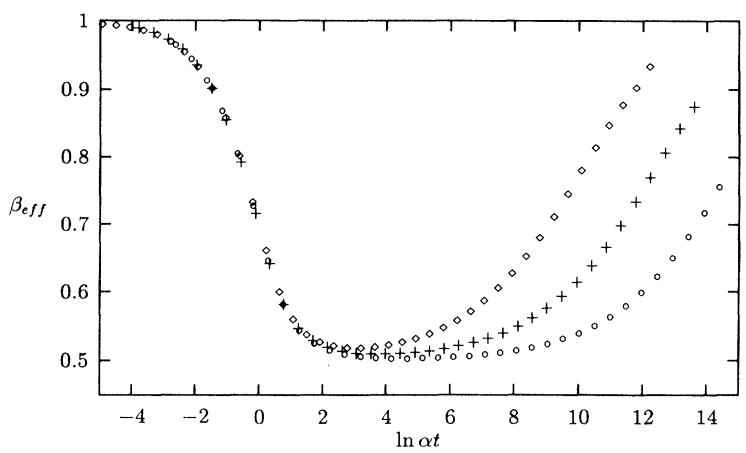

FIG. 3. Plot of the time-dependent parameter $\beta_{\text {eff }}$, defined in Eq. (58), for three different values of the temperature, namely, $\epsilon=10^{-4}$ (diamonds), $10^{-5}$ (crosses), and 10 ${ }^{-6}$ (circles). For both very short and very long times, $\beta_{\text {eff }} \rightarrow 1$, while as the temperature decreases an intermediate time window develops with $\beta_{\text {eff }}=1 / 2$. The decay from its short time value to its intermediate one is independent of the temperature, as discussed in the text.

Use of Eq. (57) yields

$$
\beta_{\mathrm{eff}}(t)=\frac{\partial}{\partial \ln t}\left\{\alpha t e^{-\alpha t}\left[I_{0}(\alpha t)+I_{1}(\alpha t)\right]\right\} .
$$

The important point is that this expression, valid for short times and in the low-temperature limit, does not depend on $\epsilon$. On the other hand, it correctly predicts the decay of $\beta_{\text {eff }}$ from its initial value $\beta_{\text {eff }}=1$ to the intermediate value $\beta_{\text {eff }}=1 / 2$. This result is confirmed by the curves plotted in Fig. 3. Note that the time window described by the KWW function, which corresponds to the horizontal part of the curves, increases as the temperature decreases. A similar behavior of $\beta_{\text {eff }}$ has been found in other models [16] and for different relaxation properties $[10,17]$. The physical origin of the general property can be roughly explained in the following way. Let $\phi(t)$ be a relaxation property verifying $\phi(0)=1$. As the temperature goes to zero, one expects the system to be frozen and therefore in the low-temperature limit and for short times we can write

$$
\phi(t) \simeq 1-\nu \xi(t)
$$

where $\nu$ is a parameter going to zero as $T \rightarrow 0$. It follows that

$$
\ln [-\ln \phi(t)] \simeq \ln \nu+\ln \xi(t)
$$

and $\beta_{\text {eff }}$ will be independent of $\nu$.

\section{CONCLUSION}

We have studied in detail the time evolution of the equilibrium spin-spin time correlation functions in the one-dimensional Ising model with Glauber dynamics. In the low-temperature limit, we have derived a continuous space equation for the evolution of these correlation 
functions and identified from it the main mechanisms of relaxation at low temperatures. For the equilibrium spin autocorrelation function, an exact Cole-Davidson behavior with $\beta_{\mathrm{CD}}=1 / 2$ is obtained in the frequency picture, while in the time domain a KWW function, with an exponent $\beta_{\mathrm{KWW}}=1 / 2$, followed by an exponential is found. The value $\beta_{\mathrm{KWW}}=0.63$ reported previously in the literature could then be considered as giving some information about the average shape of the relaxation curve of the spin time autocorrelation function, but it cannot be concluded that the relaxation in Glauber's model is less cooperative than in other systems with locally constrained dynamics.

For the initial part of the relaxation, before the KWW behavior emerges, we have shown that the spin time autocorrelation function decays very little in this time scale, for low enough temperatures. Nevertheless, there are two results concerning this regime that are worth mentioning. First, the relevant KWW function can be obtained in an intermediate time window, matching the result given by the continuous space equation. Second, the parameter $\beta_{\text {eff }}$ defined in the text decays over a universal curve in the sense that is independent of the temperature, from its short time value $\beta_{\text {eff }}=1$ to its intermediate time value $\beta_{\text {eff }}=1 / 2$. The former corresponds to the initial exponential relaxation and the latter to the KWW function. On the other hand, in this time regime we cannot obtain any information about the final exponential relaxation or, consequently, about the range of validity of the stretched exponential function.

It must also be pointed out that almost any model based on a master equation formulation leads to exponential relaxation in both the short and the long time limits, but with different characteristic time scales. Then, a KWW function appears in an intermediate time window [3]. We think that different choices of the transition rates simply shift the relevant time regimes (initial exponential, KWW function, and final exponential) to greater or smaller values of the relaxation function under consideration. The same remark remains valid in regard to the relaxation of different properties in the same model. For instance, the relaxation of the energy after an homogenous temperature perturbation can also be analyzed in Glauber's model. The continuous space equation governing the dynamics of the system at low temperatures is again Eq. (37), but the initial condition is not the one in Eq. (38), but $f(x, 0)=x \exp (-x)$ [17]. The same three time regimes appearing in Eq. (47) show up in this case, but the KWW function extends to smaller values of the relaxation function [10].

\section{ACKNOWLEDGMENT}

Partial support from Dirección General de Investigación Científica y Técnica (Spain) through Grant No. PB92-0683 is gratefully acknowledged.
[1] G. W. Scherer, Relaxation in Glass and Composites (Wiley, New York, 1986); Relaxations in Complex Systems, edited by K. L. Ngai and G. B. Wright (U.S. GPO, Washington, DC, 1985).

[2] G. H. Fredrickson, Annu. Rev. Phys. Chem. 39, 149 (1988).

[3] J. J. Brey, A. Prados, and M. J. Ruiz-Montero, J. NonCryst. Solids 172-174, 371 (1994).

[4] J. E. Anderson, J. Chem. Phys. 52, 2821 (1970).

[5] S. Bozdemir, Phys. Status Solidi B 104, 37 (1980); 103, 459 (1981).

[6] R. J. Glauber, J. Math. Phys. 4, 294 (1963).

[7] C. P. Lindsey and G. D. Patterson, J. Chem. Phys. 73, 3348 (1980).

[8] J. L. Skinner, J. Chem. Phys. 79, 1955 (1983).

[9] J. Budimir and J. L. Skinner, J. Chem. Phys. 82, 5232
(1985).

[10] J. J. Brey and A. Prados, Physica A 197, 569 (1993).

[11] H. Spohn, Commun. Math. Phys. 125, 3 (1989).

[12] G. H. Fredrickson and H. C. Andersen, Phys. Rev. Lett. 53, 1244 (1984); J. Jackle and S. Eisinger, Z. Phys. B 84, 115 (1991).

[13] N. G. van Kampen, Stochastic Processes in Physics and Chemistry (North-Holland, Amsterdam, 1981).

[14] J. E. Shore and R. Zwanzig, J. Chem. Phys. 63, 5445 (1975).

[15] The series in Eq. (29) of Ref. [9] can be easily summed. Note the difference of a factor 2 in the definition of the transition rates.

[16] P. Harrowell, Phys. Rev. E 48, 4359 (1993).

[17] J. J. Brey and A. Prados (unpublished). 